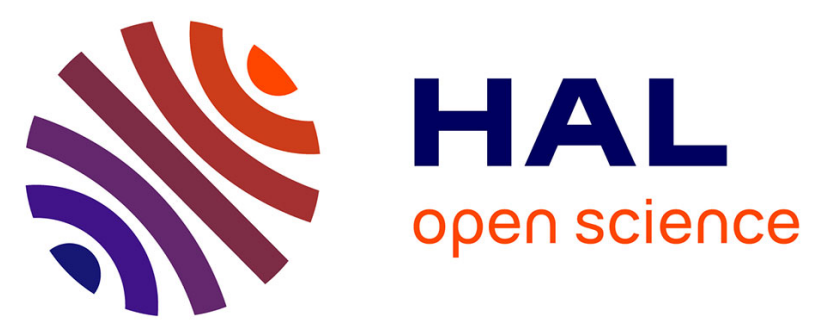

\title{
L'addiction à l'alimentation: définition, mesure et limites du concept, facteurs associés et implications cliniques et thérapeutiques
}

Sarah Cathelain, Paul Brunault, Nicolas Ballon, Christian Réveillère, Robert Courtois

\section{To cite this version:}

Sarah Cathelain, Paul Brunault, Nicolas Ballon, Christian Réveillère, Robert Courtois. L'addiction à l'alimentation: définition, mesure et limites du concept, facteurs associés et implications cliniques et thérapeutiques. La Presse Médicale, 2016, 10.1016/j.lpm.2016.03.014 . hal-01379564

\section{HAL Id: hal-01379564 https://hal.science/hal-01379564}

Submitted on 16 Nov 2016

HAL is a multi-disciplinary open access archive for the deposit and dissemination of scientific research documents, whether they are published or not. The documents may come from teaching and research institutions in France or abroad, or from public or private research centers.
L'archive ouverte pluridisciplinaire HAL, est destinée au dépôt et à la diffusion de documents scientifiques de niveau recherche, publiés ou non, émanant des établissements d'enseignement et de recherche français ou étrangers, des laboratoires publics ou privés. 
Titre : L'addiction à l'alimentation : définition, mesure et limites du concept, facteurs associés et implications cliniques et thérapeutiques

Sarah Cathelain ${ }^{1^{*}}$, Paul Brunault ${ }^{1,2,3^{*}}$, Nicolas Ballon $^{2,4}$, Christian Réveillère $^{1}$, Robert Courtois ${ }^{1,3}$

${ }^{1}$ Université François Rabelais de Tours, Département de Psychologie, EA 2114, « Psychologie des Âges de la Vie », Tours, France

${ }^{2}$ CHRU de Tours, Équipe de Liaison et de Soins en Addictologie, Tours, France

${ }^{3}$ CHRU de Tours, Clinique Psychiatrique Universitaire, Tours, France

${ }^{4}$ UMR INSERM U930 ERL, Tours, France

* Ces auteurs ont contribué de manière équivalente à ce travail

\section{Auteur correspondant :}

Paul Brunault

CHRU de Tours, 2 boulevard Tonnellé, 37000 Tours, France

e-mail : paul.brunault@univ-tours.fr

Téléphone : 02-47-47-80-43

Fax : 02-47-47-84-02

Nombre de mots: 6031 (3611 à l'exclusion des références, figures, tableaux et résumés)

This is the postprint version (final draft post refereeing) of the following article: "Sarah Cathelain, Paul Brunault, Nicolas Ballon, Christian Réveillère, Robert Courtois(2016). L'addiction à l'alimentation : définition, mesure et limites du concept, facteurs associés et implications cliniques et thérapeutiquesPresse Médicale2016 May 19. pii: S0755-4982(16)300550. doi: 10.1016/j.lpm.2016.03.014.", which has been published in final form at: http://www.emconsulte.com/en/article/1056397 


\section{Résumé des points clés :}

- Les addictions, qui se caractérisent par une impossibilité à contrôler un comportement malgré la connaissance de ses conséquences négatives, ont des déterminants biologiques, psychologiques et sociaux.

- $\quad$ S'il est désormais bien démontré qu'il est possible de développer une addiction vis-àvis de certaines substances psychoactives (i.e., alcool, tabac, cannabis)et de la pratique des jeux de hasard et d'argent (i.e., jeu pathologique) la possibilité de développer une addiction sans drogue (ou addiction comportementale) vis-à-vis d'autres comportements sources de plaisir (i.e., alimentation, conduites sexuelles, achats) est encore débattue.

- Le concept d'addiction à l'alimentation, qui fait référence à une relation de dépendance d'un individu vis-à-vis de certains aliments riches en graisse ou en sucre, a été récemment proposé en appliquant au comportement alimentaire les critères DSM de dépendance à une substance.

- Pour mesurer l'addiction à l'alimentation, la Yale Food Addiction Scale est actuellement l'auto-questionnaire de référence (diagnostic d'addiction à l'alimentation et évaluation du nombre de symptômes d'addiction à l'alimentation).

- L'addiction à l'alimentation est plus fréquente chez les patients obèses, chez les patients présentant certaines caractéristiques psychopathologiques (dépression, trouble déficitaire de l'attention avec ou sans hyperactivité, forte impulsivité), célibataires ou encore, qui présentent certaines altérations neurobiologiques du système de la récompense. Il n'est par contre pas démontré à l'heure actuelle que ce trouble soit systématiquement responsable d'une prise de poids et/ou d'une obésité.

- Un nombre croissant de travaux soulignent les proximités cliniques, neurobiologiques et en termes de facteurs de risque (biologiques, psychopathologiques, socio-culturels) entre l'addiction à l'alimentation et les autres addictions. Afin de tester la pertinence de considérer l'addiction à l'alimentation comme une addiction à part entière, il serait intéressant de réaliser des études auprès de patients pouvant présenter d'autres dommages que l'obésité: patients souffrant de syndrome métabolique, diabète de type 2, hypertension, dyslipidémie, athérosclérose, accident vasculaire cérébral ou cardiopathie ischémique.

- L'addiction à l'alimentation est un concept clinique multidimensionnel qui requiert une prise en charge intégrative impliquant des axes psychothérapeutiques, pharmacologiques et sociaux. L'intérêt de ce concept réside également dans ses implications en termes de prévention et de santé publique, via par exemple la possibilité de mesures de prévention précoces ciblées sur les patients vulnérables (information et interventions précoces, voire réglementation). 


\section{Highlights:}

- $\quad$ Addictions, which are characterized by the inability to control a behavior despite existence of physical or psychological consequences, have biological, psychological and social determinants.

- $\quad$ Although the possibility of developing an addiction to some psychoactive substances (e.g. alcohol, tobacco, cannabis) and to gambling (i.e., gambling disorder) is now well-demonstrated, the possibility to develop a non-drug addiction (i.e., behavioral addiction) to certain behaviors which provide pleasure (e.g. eating, having sex, buying things) is still in debate.

- The concept of food addiction, which refers to people who exhibit substance dependence criteria in relation to some high-fat and high-sugar foods, was recently proposed by applying substance dependence DSM criteria to eating behavior.

- $\quad$ To assess food addiction, the Yale Food Addiction Scale is now the only self-administered questionnaire (diagnosis and estimate of the number of symptoms of food addiction).

- $\quad$ Prevalence for food addiction is higher in overweight and obese patients, and in patients with certain psychopathological characteristics (i.e., depression, Attention Deficit Hyperactivity Disorder, high impulsivity), in patients who are single and in patients with neurobiological alterations in the reward system. However, it is still unclear whether food addiction is necessary associated with subsequent increase in body weight and/or obesity.

- An increasing number of studies demonstrated that drug addiction and food addiction shares some similar clinical, neurobiological and psychopathological and sociocultural risk factors. To test the pertinence to include food addiction as an addiction, it would be interesting to conduct future studies in patients who may experience harms related to their food addiction, including not only patients with obesity, but also patients with metabolic syndrome, type 2 diabetes, hypertension, dyslipidemia, atherosclerosis, stroke, or coronary heart disease.

- $\quad$ Food addiction is a clinical and multidimensional concept which requires integrated care with psychotherapy, pharmacological and social lines of approach. This concept has also practical implications in terms of prevention and public health (e.g., prevention, brief interventions, possible law enforcement regarding some kinds of food which could present some addictive properties). 


\section{1) Introduction : précisions conceptuelles addiction, addiction sans drogues, addiction à l'alimentation}

Les addictions sont des pathologies chroniques se caractérisant par une impossibilité à contrôler un comportement et une poursuite de ce comportement malgré des conséquences négatives (1). Elles sont encore au carrefour de facteurs biologiques, psychologiques et sociaux (2). Initialement, les travaux portant sur les addictions étaient centrés sur la consommation de produits tels que l'alcool, le tabac ou les substances illicites. La classification des troubles mentaux DSM-IV-TR a ainsi permis de différencier trois types de consommation de substance psychoactive : l'usage, l'abus et la dépendance (1). Plus récemment, la classification DSM-5 (3) a opéré un changement dans sa considération du trouble de l'utilisation d'une substance en passant d'une approche catégorielle à une approche dimensionnelle. Le DSM-5 permet une approche plus fine de la sévérité de l'addiction avec l'introduction du craving (envie irrépressible de consommer) comme nouveau critère clinique et avec la proposition d'un continuum de sévérité : absence d'addiction (moins de 2 critères diagnostiques sur les 11 proposés), addiction d'intensité légère (de 2 à 3 critères), addiction d'intensité modérée (4 à 5 critères) et addiction d'intensité sévère (au moins 6 critères).Selon le DSM-5, il y a addiction dès qu'il existe au moins 2 critères sur 11 et que la personne rapporte une souffrance cliniquement significative associée.

Il est désormais bien démontré qu'il est possible de développer une addiction vis-à-vis des substances psychoactives. La possibilité de développer une addiction sans drogue (aussi appelée addiction comportementale) est bien établie pour la pratique des jeux de hasard et d'argent (i.e., addiction aux jeux de hasard et d'argent ou jeu pathologique), mais reste encore débattue pour d'autres comportements sources de plaisir (i.e. alimentation, conduites sexuelles, utilisation d'Internet, achats).

Avec l'introduction du concept d'addiction comportementale (ou addiction sans drogue), il est possible de développer une addiction vis-à-vis d'une expérience ou d'un comportement qui est répété afin de procurer un plaisir ou de soulager un malaise intérieur. Le concept d'addiction comportementale a été développé suite aux travaux de Goodman, qui a proposé une définition qui reste largement utilisée : "Addiction, employée de manière descriptive, désigne la répétition d'actes susceptibles de provoquer du plaisir mais marqués par la dépendance à un objet matériel ou à une situation recherchés et consommés avec avidité »(4). Cette définition inclut les notions de dépendance, de plaisir et de répétition. Goodman a également identifié des critères communs aux addictions, face au besoin de définir des critères qui ne sont pas exclusivement liés à un objet spécifique de dépendance (5). Parmi ces critères, deux sont majeurs dans le mécanisme addictif à l'œuvre dans les addictions comportementales: l'impossibilité de résister aux impulsions à réaliser ce type de comportement, et la poursuite du comportement bien que le sujet sache qu'il cause ou aggrave un problème persistant ou récurrent d'ordre social, financier, psychologique ou physique. La particularité des addictions comportementales est la relation spécifique entre une personne et un objet sans toxicité apparente, largement utilisé et qui n'est pas source de sanction sociale s'il est utilisé, tels que la pratique de jeux de hasard et d'argent, le fait de manger, ou de réaliser des activités comme le travail ou le sport (6). Si la limite du champ des addictions comportementales (ou addictions sans drogue) est encore débattue, son existence est désormais bien reconnue dans les classifications internationales, notamment avec l'introduction de l'addiction aux jeux de hasard et d'argent (i.e., jeu pathologique) dans le DSM-5. Les débats actuels consistent à savoir s'il est possible de développer une addiction visà-vis d'autres comportements sans toxicité apparente (i.e., pratique des jeux des jeux vidéo, activités sexuelles, usage d'internet, achats, exercice physique). Les réponses à ces débats permettront de savoir si ces troubles peuvent être considérés ou non comme des addictions, et si oui, quelles en seraient les prises en charge optimales.

Bien que l'alimentation soit facilement accessible et largement disponible dans nos sociétés occidentalisées,les travaux examinant la possibilité de développer une addiction vis-à-vis de 
l'alimentation ne se sont développés que récemment (7-9). Les débats autour de cette question demeurent très animés, notamment car l'alimentation est fondamentale à notre survie, alors à partir de quels critères peut-on définir la dépendance à l'alimentation ? Comment parler de dépendance pour un objet qui est nécessaire à notre survie, et auquel nous ne pouvons pas renoncer ?

\section{2) Le concept d'addiction à l'alimentation : comment le définir ? comment le mesurer?}

\section{a) Définition du concept}

Le concept d'addiction à l'alimentation fait référence aux critères diagnostiques DSM de dépendance à une substance. Ce concept d'apparition récente (10) vise à décrire les difficultés cliniques que rencontrent certains patients dans leur relation à l'alimentation: la perte de contrôle sur la consommation alimentaire, une incapacité à réduire leur consommation malgré le désir de le faire ou encore la poursuite de comportement malgré la connaissance des effets négatifs de cette consommation alimentaire sur leur santé (11). Ashley Gearhardt et son équipe de recherche ont proposé le concept d'addiction à l'alimentation, et son opérationnalisation avec le premier outil permettant de le mesurer : la Yale Food Addiction Scale(7). Différents arguments neurobiologiques, comportementaux et cliniques ont été avancés dans la littérature scientifique en faveur de l'intégration du concept d'addiction à l'alimentation dans le champ des addictions (pour une revue de la question, voir 12).

\section{b) Mesure du concept}

L'auto-questionnaire Yale Food Addiction Scale est actuellement l'outil de référence pour évaluer cette addiction (7). Cet outil a été développé dans le but de fournir un outil normalisé pour évaluer les symptômes d'addiction à l'alimentation, spécifiquement par rapport à certains aliments gras, salés ou sucrés, pouvant avoir des propriétés addictives (7). Cette échelle est composée de 25 items (de type Likert ou dichotomiques), basés sur les sept critères de dépendance à une substance du DSM-IV-TR, et se rapportant aux 12 derniers mois. Cet outil d'évaluation qui permet d'évaluer l'addiction à l'alimentation de deux manières: 1) le nombre de symptômes d'addiction à l'alimentation présents au cours des 12 derniers mois et 2) diagnostic éventuel d'addiction à l'alimentation. Les recherches actuelles ont démontré les très bonnes propriétés psychométriques de cet outil (très bonne validité et fiabilité, structure à un facteur témoignant de la pertinence d'utiliser un score global plutôt que plusieurs scores).

La YFAS a été traduite en allemand (13) et en français (14). La version française est libre de droit (14). Une version plus courte («version épidémiologique ») a également été proposée : la YFASmodifiée (mYFAS) qui est composée de 9 items dont 7 évaluant chacun des sept symptômes de dépendance à une substance du DSM-IV-TR, et deux évaluant la présence d'une souffrance cliniquement significative (15). Cette version courte anglaise (15) a été adaptée en français par S. Berthoz, A. Carré, J. Swendsen et C. Dantzer(16), cette dernière étant présentée en Figure 1. Il existe aussi une version pour enfant (YFAS-C), adaptée afin de faire référence à des activités en lien avec l'âge et le niveau de lecture des enfants et pour laquelle les catégories de réponse ont été reformulées afin d'en faciliter la compréhension (17).

\section{c) Prévalence de l'addiction à l'alimentation et facteurs associés}

Les études utilisant l'échelle YFAS ont mis en évidence une prévalence de 5 à $10 \%$ d'addiction à l'alimentation en population non clinique (18). Les prévalences sont plus élevées dans les échantillons de patients obèses (entre 15 et $25 \%$ ), et le diagnostic d'addiction à l'alimentation est plus fréquent (plus de 50\%) chez les personnes ayant des troubles du comportement alimentaire (bingeeatingdisorder $=$ hyperphagie boulimique, boulimie nerveuse, et dans une moindre mesure, anorexie mentale) (19). Le critère d'addiction à l'alimentation le plus fréquemment constaté en 
population générale et chez les patients est «le désir persistant ou des efforts infructueux pour diminuer ou contrôler la consommation » (15). Les autres symptômes les plus souvent mentionnés sont la poursuite de la consommation de certains aliments en dépit de problèmes physiques ou psychologiques et la tolérance, notamment chez des personnes obèses (20).

Dans la littérature, différents travaux ont démontré des associations statistiques entre l'addiction à l'alimentation et plusieurs types de facteurs; nous différencierons ici les facteurs individuels, les facteurs biologiques, les facteurs psychopathologiques et les facteurs environnementaux (pour une revue de la littérature détaillée, voir Brunault et al., 12)). Les facteurs associés à l'addiction à l'alimentation sont résumés dans la Figure 2.

\section{- $\quad$ Facteurs individuels}

Les personnes présentant une addiction à l'alimentation étaient plus fréquemment célibataires, de sexe féminin, plus âgée, et rapportaient plus fréquemment une altération de l'estime de soi $(20,21)$. Elles consommaient davantage certains types d'aliments (gras, sucrés) dans des contextes où elles ressentaient des émotions négatives (22). Il a été démontré que l'impulsivité attentionnelle, des difficultés de mentalisation et des difficultés de régulation émotionnelle étaient également associées à l'addiction à l'alimentation $(21,23)$.

\section{- $\quad$ Facteurs biologiques}

Les personnes présentant une addiction à l'alimentation étaient plus fréquemment obèses, avaient un poids et un IMC moyen plus élevés (15). Parmi les patients obèses, il n'est par contre pas démontré que l'addiction à l'alimentation soit nécessairement associée à une obésité plus sévère. L'addiction à l'alimentation était également associée à des facteurs neurobiologiques (activation plus forte du circuit de la récompense et moindre activation des régions inhibitrices (24)).

\section{- Facteurs psychopathologiques}

L'addiction à l'alimentation était associée à des scores de dépression plus importants, aux symptômes de stress post-traumatique, et aux symptômes d'hyperactivité/inattention dans l'enfance $(21,23,25)$. L'addiction à l'alimentation était également associée à des épisodes de craving plus intenses et plus fréquents (11), ainsi qu'aux comportements boulimiques (avec ou sans vomissement) (10), au bingeeatingdisorder(21) et à la boulimie nerveuse et dans une moindre mesure à l'anorexie mentale (26).

\section{- Facteurs liés à certaines caractéristiques de l'environnement}

Les personnes présentant une addiction à l'alimentation consommaient davantage et en quantités plus importantes les aliments transformés, et les aliments riches en graisse, en sel et/ou en sucre (27). Des antécédents de traumatismes physiques ou sexuels sont plus fréquemment constatés chez les patients souffrant d'addiction à l'alimentation.

\section{3) Discussion autour du concept d'addiction à l'alimentation}

\section{a) Arguments en défaveur du concept}

Selon certains auteurs, le concept d'addiction à l'alimentation est pour l'instant largement débattu et controversé, notamment en raison de difficultés de définition de cette addiction et d'un manque actuel de données scientifiques sur le sujet (23). Pour ces auteurs qui remettent en question la pertinence du concept, les modèles existants ne peuvent aller au-delà de la relative dépendance à des catégories d'aliments à haute teneur en graisse, sucre ou des aliments agréables au goût. Bien que ces aliments soient nocifs pour la santé d'un point de vue métabolique et cardiovasculaire, il n'y a pas de données actuelles concernant la concentration particulière de nutriments qui pourraient engendrer un 
processus addictif (28). Ainsi, une des difficultés de la notion d'addiction à l'alimentation est de déterminer quels types d'aliments pourraient avoir des propriétés addictives, via des critères scientifiques (ou objectivables) rigoureux. Toujours selon ces auteurs, le concept prétendument mesuré à l'aide de l'échelle YFAS pourrait n'être en fait que le reflet d'un comportement alimentaire désorganisé plutôt qu'un comportement en lien avec un véritable processus addictif. Le risque pourrait alors être de pathologiser un comportement qui ne pourrait être que le reflet d'habitudes comportementales. De même, il est peu probable que l'addiction à l'alimentation puisse expliquer la plupart des situations d'obésité car l'obésité est un syndrome fortement hétérogène et multifactoriel. Il existe également des limites relatives à l'outil de mesure (questionnaire YFAS) : l'utilisation de mesures dichotomiques pour évaluer certains critères diagnostiques d'addiction à l'alimentation, le fait qu'il s'agisse d'un auto-questionnaire (donc surévaluant potentiellement la prévalence du trouble). Enfin, le fait que les auteurs qui sont à l'origine du concept soient également ceux qui ont proposé cet outil de mesure peut être considéré comme une limite.

\section{b) Arguments favorables au concept}

De nombreux travaux présentent un lien entre certains troubles des conduites alimentaires comme la consommation excessive de nourriture ou le bingeeatingdisorder (aussi appelée hyperphagie boulimique) et un comportement addictif (10). Il semblerait que le bingeeatingdisorder ait des similitudes comportementales et neurobiologiques avec les addictions aux drogues, ce qui renforce la possibilité que certains des troubles du comportement alimentaire puissent être liés à une addiction à l'alimentation (23). Ces conduites concernent principalement les aliments industriels, qui sont riches en sucre, en graisse ou en sel $(9,11,29)$. Ainsi, certains types d'aliments, tels que les aliments raffinés, pourraient présenter des propriétés addictives comparables à celles retrouvées dans l'alcool, le tabac ou encore la cocaïne (30). En France, l'équipe de Serge Ahmed a été une des premières à étudier l'addiction à l'alimentation. En se basant sur des modèles animaux, cette équipe a ainsi démontré que l'addiction à l'alimentation, et plus particulièrement l'addiction aux produits sucrés riches en sucres raffinés (ex., saccharose, fructose) était associée à des altérations fonctionnelles des circuits neurobiologiques impliqués dans l'apprentissage de la récompense, la motivation et le contrôle de la récompense, ces altérations étant proches de celles observées dans l'addictions aux drogues (ex., cocaïne) (9). D'autres auteurs ont proposé l'hypothèse complémentaire d'une addiction aux produits salés qui pourrait expliquer pour partie l'augmentation de prévalence d'obésité dans nos sociétés occidentalisées (29). Ces travaux suggèrent l'existence de différents sous-types d'addiction à l'alimentation (addiction aux produits sucrés riches en sucres raffinés vs. addiction aux produits salés), dont nous pouvons faire l'hypothèse que certains dommages soient communs (surpoids et obésité) tandis que d'autres seraient plus spécifiques d'un sous-type d'addiction (diabète de type 2 pour l'addiction au sucre vs. hypertension pour l'addiction au sel).

Les études animales ont établi un parallèle entre la consommation excessive de nourriture et la dépendance à une substance (30). Les rats ayant un accès facilité aux aliments riches en sucre, graisse et aux aliments transformés présentaient des altérations neurobiologiques similaires à celles retrouvées dans la dépendance à une substance (9). Toujours chez l'animal, ces chercheurs ont également démontré l'existence de symptômes de sevrage et de tolérance vis-à-vis de certains aliments (30).

Les travaux de neuro-imagerie ont permis d'identifier que la consommation des aliments hautement palatables activait les mêmes systèmes neurobiologiques que ceux impliqués dans la consommation de substances psychoactives (31). Les patients ayant des scores d'addiction à l'alimentation plus élevés présentaient une altération du circuit de la récompense comparable à celle observée dans les autres addictions : plus forte activation du cortex préfrontal dorso-latéral et du noyau caudé en réponse à l'anticipation de la prise alimentaire, moindre activation du cortex orbito-frontal latéral en réponse à la prise alimentaire (24). Une des similitudes les plus convaincantes entre 
l'addiction à l'alimentation et l'addiction aux substances psychoactives est l'expérience du craving, qui est régulièrement accompagnée d'une perte de contrôle et d'une prise d'aliments ou de substance (11). L'activation de l'insula, qui est fréquemment impliquée dans la consommation de substances (32), est aussi impliquée dans les symptômes de sevrage vis-à-vis de certains aliments et lors de périodes de fort craving pour l'alimentation (33).

\section{4) Application clinique dans le domaine de l'obésité}

Si l'obésité est une maladie multifactorielle complexe, les travaux épidémiologiques ont démontré l'augmentation récente de la prévalence de ce trouble dans les pays occidentalisés. Selon certains auteurs, les troubles du comportement alimentaire ou l'addiction à l'alimentation pourraient être, en complément des prédispositions génétiques et des facteurs environnementaux, parmi les principaux facteurs responsables de cette prévalence croissante d'obésité (34). L'addiction à l'alimentation étant plus fréquente chez les personnes obèses, nous pouvons supposer que l'addiction à l'alimentation puisse contribuer, chez certains patients, à la constitution ou au maintien de l'obésité. Selon cette hypothèse, il serait nécessaire de prendre en charge ce trouble chez cette sous population de patients afin de limiter le risque d'aggravation ou de maintien de l'obésité. Ainsi, poursuivre l'étude des déterminants de l'addiction à l'alimentation apporterait de nouveaux éléments de compréhension dans la prise en charge de certains patients obèses.

\section{5) Application clinique auprès d'autres patients}

Différentes études présentent un lien entre certains troubles du comportement alimentaire (bingeeatingdisorder ou hyperphagie boulimique, boulimie nerveuse) et un comportement addictif. Selon les classifications internationales, il est nécessaire que le critère « consommation d'une quantité d'aliments largement supérieure à ce que d'autres personnes pourraient consommer dans la même période de temps » soit rempli pour diagnostiquer un trouble du comportement alimentaire de type hyperphagie boulimique ou boulimie nerveuse, avec cette notion de quantité de consommation et de fréquence. Toutefois, certains patients peuvent rapporter des difficultés à contrôler leur alimentation sans pour autant que ce critère ne soit rempli. À l'instar de la dépendance à l'alcool qui ne se définit pas par une quantité d'alcool consommé, la quantité d'aliments consommée n'est pas un critère nécessaire au diagnostic d'addiction à l'alimentation. Le concept d'addiction à l'alimentation permet de mieux appréhender les difficultés que certains individus (qui ne remplissent pas les critères de bingeeatingdisorder ou de boulimie nerveuse) peuvent rencontrer sur le plan alimentaire.

Si la plupart des travaux ont porté chez les patients obèses, l'addiction à l'alimentation peut également être associée à d'autres dommages physiques: la forte prévalence d'addiction à l'alimentation chez les patients diabétiques (70\% selon l'étude de Raymond et al. (32)) suggère qu'il est possible que cette addiction s'accompagne d'autres dommages. De même, il serait intéressant de réaliser des études auprès de patients pouvant présenter d'autres dommages que l'obésité : patients souffrant de syndrome métabolique, diabète de type 2, hypertension, dyslipidémie, athérosclérose, accident vasculaire cérébral ou cardiopathie ischémique. S'il pouvait être démontré que l'addiction à l'alimentation s'accompagnait d'autres dommages, cela constituerait un argument en faveur de la pertinence d'un modèle addictif du comportement alimentaire.

\section{6) Restriction cognitive et addiction à l'alimentation : quelles spécificités thérapeutiques?}

La restriction cognitive se définit comme l'intention de maitriser son poids par un contrôle volontaire et planifié du comportement alimentaire (35). Cela inclut par exemple le fait de s'interdire certains aliments, avec une alternance entre contrôle et perte de contrôle sur le comportement alimentaire. La restriction cognitive est classiquement un facteur de risque de trouble du comportement alimentaire et a un impact sur l'estime de soi (majoration du sentiment de culpabilité en 
cas d'échec de contrôle). Bien l'on ne connaisse pas encore la nature des liens entre addiction à l'alimentation et restriction cognitive, nous pouvons supposer que la restriction cognitive soit plus importante chez ces patients. Il est donc nécessaire de rechercher systématiquement cette dimension et de l'intégrer à part entière dans la stratégie thérapeutique. Face à un patient ayant une addiction à l'alimentation et une forte restriction cognitive, la demande du patient est fréquemment motivée par une perte de poids. Un des enjeux est de trouver un point d'équilibre entre la tolérance et la contrainte, l'astreinte (la permanence du traitement) d'une part et l'idéal de perte de poids d'autre part. Dans ce cas, l'objectif thérapeutique ne se limitera pas à la seule perte de poids ou au seul contrôle de l'addiction: il conviendra de chercher de manière plus générale à restituer au comportement alimentaire ses finalités nutritionnelles et relationnelles à l'aide d'une approche intégrative (35).

\section{7) Implications thérapeutiques}

L'aspect multidimensionnel de l'addiction à l'alimentation souligne l'intérêt d'une prise en charge intégrative qui ne se limite pas à sa dimension biologique ou psychologique stricte. Du point de vue thérapeutique, trois axes d'intervention sont possibles : psychothérapeutique, pharmacologique et social. En ce qui concerne l'axe psychothérapeutique, on retrouve l'intérêt des approches motivationnelles et des psychothérapies, notamment celles centrées sur les facteurs de vulnérabilité psychopathologiques du patient (ex., impulsivité, comorbidités psychiatriques, régulation émotionnelle, alexithymie). Pour ce qui est de l'axe pharmacologique, certains traitements efficaces dans le champ des addictions (i.e., traitements ciblant le système de la récompense et les circuits opioïdes, GABAergiques, glutamatergiques ou dopaminergiques) pourraient être efficaces pour prendre en charge l'addiction à l'alimentation. Rappelons néanmoins qu'à ce jour, cette hypothèse est purement spéculative et qu'aucun traitement médicamenteux n'a d'Autorisation de Mise sur le Marché dans l'indication "addiction à l'alimentation ». Enfin, dans l'axe social, on retrouve l'intérêt d'une prise en charge de groupe (groupes d'entraide) et d'une approche préventive, avec un objectif de réduction des risques. En proposant une vision biopsychosociale de l'addiction à l'alimentation, il est également possible de contribuer à une déstigmatisation de ces patients, qui présentent souvent une culpabilité importante associée à leur comportement alimentaire. Enfin, l'opérationnalisation de la mesure du concept d'addiction à l'alimentation permet de tester l'hypothèse de transitions entre addictions chez des patients présentant des facteurs de risque communs aux addictions. Si cette hypothèse était vérifiée, cela signifierait qu'il faudrait systématiquement repérer et prendre en charge les autres addictions chez ces patients avant prise en charge et après prise en charge (i.e., risque potentiellement plus élevé de transfert d'addiction chez des patients pris en charge pour une addiction avec ou sans drogue).

Si le concept d'addiction à l'alimentation ne peut pas expliquer à lui seul l'obésité ou les troubles du comportement alimentaire, il peut être utile pour identifier parmi eux un sous-groupe de patients pour lesquels il est possible de proposer des prises en charge classiquement efficaces pour prendre en charge d'autres addictions (entretiens motivationnels, prévention de la rechute, psychothérapies, traitements médicamenteux ciblant les systèmes neurobiologiques impliqués dans les addictions, voire éventuellement traitements de substitution). Des études ultérieures sont nécessaires pour déterminer quelles sont les thérapeutiques susceptibles d'améliorer la prise en charge et de la qualité de vie de ces patients. Ce concept peut également avoir un impact en termes de prévention et de santé publique. S'il pouvait être démontré que l'addiction à l'alimentation était dépendante de l'accessibilité et de la disponibilité à certains aliments, une autre implication pourrait être la possibilité d'une politique de réduction des risques et d'une réflexion plus globale sur la place de l'alimentation dans nos sociétés occidentalisées, voire même d'une réglementation vis-à-vis de la commercialisation de certains aliments, semblable aux réglementations sur le tabac ou l'alcool. 


\section{Bibliographie}

1. American Psychiatric Association. Diagnostic and statistical manual of mental disorders: DSM-IV-TR. 4 ed. Washington, DC, US: American Psychiatric Association; 2000.

2. Olievenstein C. La drogue ou la vie. Paris, FR: Éditions Robert Laffont; 1986.

3. American Psychiatric Association. Diagnostic and Statistical Manual of Mental Disorders, Fifth Edition. New-York, NY, US: American Psychiatric Press; 2013.

4. Goodman A. Addiction: definition and implications. Br J Addiction. 1990;85(11):1403-8.

5. Les addictions sans drogue(s) [Internet]. Toxibase. 2002. Available from: http://fulltext.bdsp.ehesp.fr/Toxibase/RevueToxibase/2002/6/01.pdf.

6. Varescon I. Les addictions comportementales: aspects cliniques et psychopathologiques. Bruxelles, BE: Editions Mardaga; 2009.

7. Gearhardt AN, Corbin WR, Brownell KD. Preliminary validation of the Yale Food Addiction Scale. Appetite. 2009;52(2):430-6.

8. Davis C, Carter JC. Compulsive overeating as an addiction disorder. A review of theory and evidence. Appetite. 2009;53(1):1-8.

9. Ahmed S. Sucres, addiction et obésité. Obésité. 2012;7(1):3-9.

10. Gearhardt AN, Corbin WR, Brownell KD. Food Addiction: An Examination of the Diagnostic Criteria for Dependence. J Addict Med. 2009;3(1):1-7.

11. Meule A, Kübler A. Food cravings in food addiction: The distinct role of positive reinforcement. Eat Behav. 2012;13(3):252-5.

12. Brunault $\mathrm{P}$. Facteurs psychopathologiques associés à la dépression, à la qualité de vie et à l'addiction à l'alimentation dans le cadre de la maladie chronique à partir du modèle de Wilson et Cleary. In: Paul Brunault (Thèse de doctorat en psychologie). L'addiction à l'alimentation: délimitation, mesure du concept et étude de ses déterminants potentiels chez les patients obèses à partir du modèle de Wilson et Cleary. Tours, FR: Université François Rabelais 2015. p. 175-244

13. Meule A, Vögele C, Kübler A. German translation and validation of the Yale Food Addiction Scale. Diagnostica. 2012;58(3):115-26.

14. Brunault P, Ballon N, Gaillard P, Réveillère C, Courtois R. Validation of the French Version of the Yale Food Addiction Scale: An Examination of Its Factor Structure, Reliability, and Construct Validity in a Nonclinical Sample. CanJPsychiatry. 2014;59(5):276-84.

15. Flint AJ, Gearhardt AN, Corbin WR, Brownell KD, Field AE, Rimm EB. Food addiction scale measurement in 2 cohorts of middle-aged and older women. Am J Clin Nutr. 2014;99(3):578-86.

16. Bourdier L, Carré A, Romo L, Dantzer C, Berthoz S. Is emotional eating the missing link between stress and food addiction? 3rd International Conference on Behavioral Addictions, Geneva, 14-16 March 2016. Abstract $n^{\circ}$ JBADD-D-15-00126 published in the J Behav Addict. 2016;5(Suppl. 1):7.doi: 10.1556/JBA.5.2015.Suppl.1

17. Gearhardt AN, Roberto CA, Seamans MJ, Corbin WR, Brownell KD. Preliminary validation of the Yale Food Addiction Scale for children. Eat Behav. 2013;14(4):508-12.

18. Lent MR, Eichen DM, Goldbacher E, Wadden TA, Foster GD. Relationship of food addiction to weight loss and attrition during obesity treatment. Obesity. 2014;22(1):52-5.

19. Pursey KM, Stanwell P, Gearhardt AN, Collins CE, Burrows TL. The Prevalence of Food Addiction as Assessed by the Yale Food Addiction Scale: A Systematic Review. Nutrients. 2014;6(10):4552-90.

20. Gearhardt AN, White MA, Masheb RM, Grilo CM. An examination of food addiction in a racially diverse sample of obese patients with binge eating disorder in primary care settings. Compr Psychiatry. 2013;54(5):500-5.

21. Gearhardt AN, White MA, Masheb RM, Morgan PT, Crosby RD, Grilo CM. An examination of the food addiction construct in obese patients with binge eating disorder. Int J Eat Disord. 2012;45(5):657-63. 
22. Boggiano MM, Burgess EE, Turan B, Soleymani T, Daniel S, Vinson LD, et al. Motives for eating tasty foods associated with binge-eating. Results from a student and a weight-loss seeking population. Appetite. 2014;83:160-6.

23. Meule A, Gearhardt AN. Five years of the Yale Food Addiction Scale: Taking stock and moving forward. Curr Addict Rep. 2014;1(3):193-205.

24. Gearhardt AN, Yokum S, Orr P, Stice E, Corbin W, Brownell K. Neural correlates of food addiction. Arch Gen Psychiatry. 2011;68(8):808-16.

25. Mason SM, Flint AJ, Roberts AL, Agnew-Blais J, Koenen KC, Rich-Edwards JW. Posttraumatic Stress Disorder Symptoms and Food Addiction in Women by Timing and Type of Trauma Exposure. JAMA Psychiatry. 2014;71(11):1271-8.

26. Granero R, Hilker I, Agüera Z, Jiménez-Murcia S, Sauchelli S, Islam MA, et al. Food Addiction in a Spanish Sample of Eating Disorders: DSM-5 Diagnostic Subtype Differentiation and Validation Data. Eur Eat Disord Rev. 2014;22(6):389-96.

27. Schulte EM, Avena NM, Gearhardt AN. Which Foods May Be Addictive? The Roles of Processing, Fat Content, and Glycemic Load. PLoS ONE. 2015;10(2):e0117959.

28. Ziauddeen H, Fletcher PC. Is food addiction a valid and useful concept? Obesity Reviews. 2013;14(1):1928.

29. Cocores JA, Gold MS. The Salted Food Addiction Hypothesis may explain overeating and the obesity epidemic. Med Hypotheses. 2009;73(6):892-9.

30. Avena NM, Rada P, Hoebel BG. Evidence for sugar addiction: Behavioral and neurochemical effects of intermittent, excessive sugar intake. Neurosci Biobehav Rev. 2008;32(1):20-39.

31. Volkow ND, Wang G-J, Fowler JS, Telang F. Overlapping neuronal circuits in addiction and obesity: evidence of systems pathology. Philos Trans R Soc Lond B Biol Sci. 2008;363(1507):3191-200.

32. Raymond K-L, Lovell GP. Food addiction symptomology, impulsivity, mood, and body mass index in people with type two diabetes. Appetite. 2015;95:383-9.

33. Pelchat ML, Johnson A, Chan R, Valdez J, Ragland JD. Images of desire: food-craving activation during fMRI. Neuroimage. 2004;23(4):1486-93.

34. Fortuna JL. The Obesity Epidemic and Food Addiction: Clinical Similarities to Drug Dependence. J Psychoact Drugs. 2012;44(1):56-63.

35. Apfeldorfer G, Zermati J. [Cognitive restraint in obesity. History of ideas, clinical description]. Presse Med. 2001;30(32):1575-80. 
Figure 1. Version courte de la Yale Food Addiction Scale (mYFAS) : adaptation française de S.

Berthoz, A. Carré, J. Swendsen, C. Dantzer) (Bourdier et al. 2015).

Ce questionnaire porte sur vos habitudes alimentaires de l'année passée.

Les gens ont parfois des difficultés à contrôler leur consommation de certains aliments tels que :

- les aliments sucrés comme la crème glacée, le chocolat, les beignets, les paquets de biscuits, les gâteaux et les bonbons.

- les féculents comme le pain blanc, les petits pains, les pâtes et le riz.

- les aliments salés comme les chips, les bretzels et les biscuits.

- les aliments gras comme le steak, le bacon, les hamburgers, les cheeseburgers, les pizzas et les frites.

- les boissons sucrées comme le soda.

Les questions suivantes portent sur ces aliments ou n'importe quel(s) autre(s) aliment(s) avec lesquels vous avez pu rencontrer des difficultés durant les 12 derniers mois.

Pour chaque question, merci d'entourer le chiffre correspondant à votre réponse.

\begin{tabular}{|c|c|c|c|c|c|}
\hline & Jamais & $\begin{array}{l}\text { Une fois } \\
\text { par } \\
\text { mois }\end{array}$ & $\begin{array}{c}2 \text { à } 4 \\
\text { fois par } \\
\text { mois }\end{array}$ & $\begin{array}{l}2 \text { à } 4 \text { fois } \\
\text { par } \\
\text { semaine }\end{array}$ & $\begin{array}{c}\text { Plus de } 4 \\
\text { fois par } \\
\text { semaine } \\
\text { ou tous les } \\
\text { jours } \\
\end{array}$ \\
\hline $\begin{array}{l}\text { 1. J'ai constaté que je continuais à consommer certains } \\
\text { aliments même lorsque je n'avais plus faim. }\end{array}$ & D & 1 & $\square$ & $\not B$ & $\square$ \\
\hline $\begin{array}{l}\text { 2. Le fait de réduire ma consommation de certains } \\
\text { aliments m'a préoccupé(e), tracassé(e). }\end{array}$ & ב & 1 & $\square$ & $\square$ & $\square$ \\
\hline $\begin{array}{l}\text { 3. Je me suis senti(e) épuisé(e) ou ramolli(e) d'avoir } \\
\text { trop mangé. }\end{array}$ & סם & $\square_{1}$ & $\square$ & $\mathbb{B}$ & $\square$ \\
\hline $\begin{array}{l}\text { 4. J'ai passé du temps à faire face aux émotions } \\
\text { négatives liées au fait d'avoir trop mangé certains } \\
\text { aliments, plutôt que de passer du temps à faire } \\
\text { d'autres activités importantes comme être avec ma } \\
\text { famille ou mes amis, travailler ou avoir des loisirs. }\end{array}$ & D & $\square 1$ & 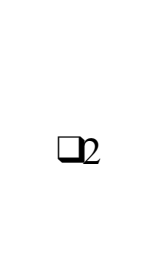 & $\not B$ & 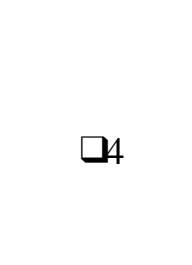 \\
\hline $\begin{array}{l}\text { 5. J'ai ressenti des symptômes de sevrage comme de } \\
\text { l'agitation, de l'anxiété lorsque j'ai diminué ou arrêté } \\
\text { de consommer certains aliments } \\
\text { (NE PAS tenir compte des symptômes dus à une } \\
\text { réduction ou un arrêt de boissons contenant de la } \\
\text { caféine: café, thé, boissons gazeuses ou énergisantes, } \\
\text { etc...). }\end{array}$ & בם & $\square 1$ & 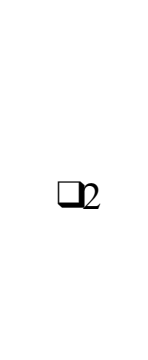 & $\square$ & $\square$ \\
\hline $\begin{array}{l}\text { 6. J'ai continué de consommer le même type ou la } \\
\text { même quantité de nourriture, en dépit de difficultés } \\
\text { émotionnelles (par exemple : dépression, de l'anxiété, } \\
\text { de la culpabilité, de la honte ou du dégoût de moi- } \\
\text { même) ou de problèmes physiques importants liés à } \\
\text { mon alimentation. }\end{array}$ & D & D1 & R & $\square$ & 4 \\
\hline $\begin{array}{l}\text { 7. J'ai constaté que de manger la même quantité de } \\
\text { nourriture ne me permet plus de réduire les émotions } \\
\text { négatives ou d'augmenter le sentiment de plaisir } \\
\text { comme cela était le cas auparavant. }\end{array}$ & 口 & D1 & $\square$ & $\not B$ & $\square$ \\
\hline
\end{tabular}




\begin{tabular}{|c|c|c|c|c|c|}
\hline & Jamais & $\begin{array}{l}\text { Une fois } \\
\text { par } \\
\text { mois }\end{array}$ & $\begin{array}{c}2 \text { à } 4 \\
\text { fois par } \\
\text { mois }\end{array}$ & $\begin{array}{l}2 \text { à } 4 \text { fois } \\
\text { par } \\
\text { semaine }\end{array}$ & $\begin{array}{c}\text { Plus de } 4 \\
\text { fois par } \\
\text { semaine } \\
\text { ou tous les } \\
\text { jours }\end{array}$ \\
\hline $\begin{array}{l}\text { 8. J'éprouve une importante détresse en raison de mon } \\
\text { rapport à la nourriture et de mon comportement } \\
\text { alimentaire. }\end{array}$ & D & $\square 1$ & $\square$ & $\not B$ & $\square$ \\
\hline $\begin{array}{l}\text { 9. J'éprouve d'importantes difficultés à fonctionner } \\
\text { efficacement (par exemple : au travail ou à l'école, } \\
\text { dans mes activités quotidiennes, en famille ou en } \\
\text { situation sociale), ou j'ai des problèmes de santé en } \\
\text { raison de mon rapport à la nourriture ou de mon } \\
\text { comportement alimentaire. }\end{array}$ & 0 & $\square 1$ & $\square$ & $\square$ & 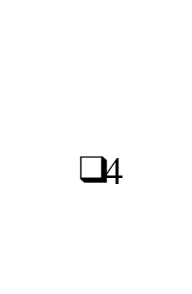 \\
\hline
\end{tabular}

QL1. Merci d'encercler TOUS les aliments avec lesquels vous avez actuellement des difficultés.

$\begin{array}{llll}\text { Crèmes glacées } & \text { Chocolat } & \text { Pommes } & \text { Beignet } \\ \text { Brocoli } & \text { Paquets de biscuits } & \text { Gâteaux } & \text { Pain blanc } \\ \text { Les petits pains } & \text { Laitue } & \text { Pâtes } & \text { Fraises } \\ \text { Riz } & \text { Crackers } & \text { Bretzels } & \text { Frites } \\ \text { Carottes } & \text { Steak } & \text { Bananes } & \text { Bacon } \\ \text { Hamburgers } & \text { Pizzas } & \text { Soda } & \text { Esquimau } \\ \text { Bonbons } & \text { Chips } & \text { Cheeseburgers } & \text { Aucun de ces aliments }\end{array}$

QL2. Merci de lister ici quels sont les autres aliments avec lesquels vous avez des difficultés (merci de ne mentionner que les aliments n'étant pas déjà dans la liste ci-dessus).

\section{Modalités de cotation de la mYFAS :}

- Les items 1 à 7 évaluent chacun des 7 critères DSM-IV-TR de dépendance à une substance (pour les items 1 et 2 : critère présent si score $\geq 4$; pour les items 3 à $5:$ critère présent si score $\geq 3$; pour les items 6 et 7 : critère présent si score $\geq 1$ ).

- Les items 8 et 9 évaluent la souffrance significative associée (critère présent si score $\geq 3$ à au moins un de ces deux items)

- Le nombre de symptômes d'addiction à l'alimentation correspond au nombre de critères présents sur les 7 possibles (score de 0 à 7 ).

- $\quad$ Il existe une addiction à l'alimentation s'il existe :

- Au moins 3 critères sur 7 d'addiction à l'alimentation (items 1 à 7)

- ET que le critère de détresse émotionnelle est présent (items 8 et 9).

- Les questions 10 et 11 permettent d'avoir une idée des types d'aliments dont il est difficile de contrôler la consommation. 
Figure2. Facteurs associés à l'addiction à l'alimentation selon les données actuelles de la littérature.

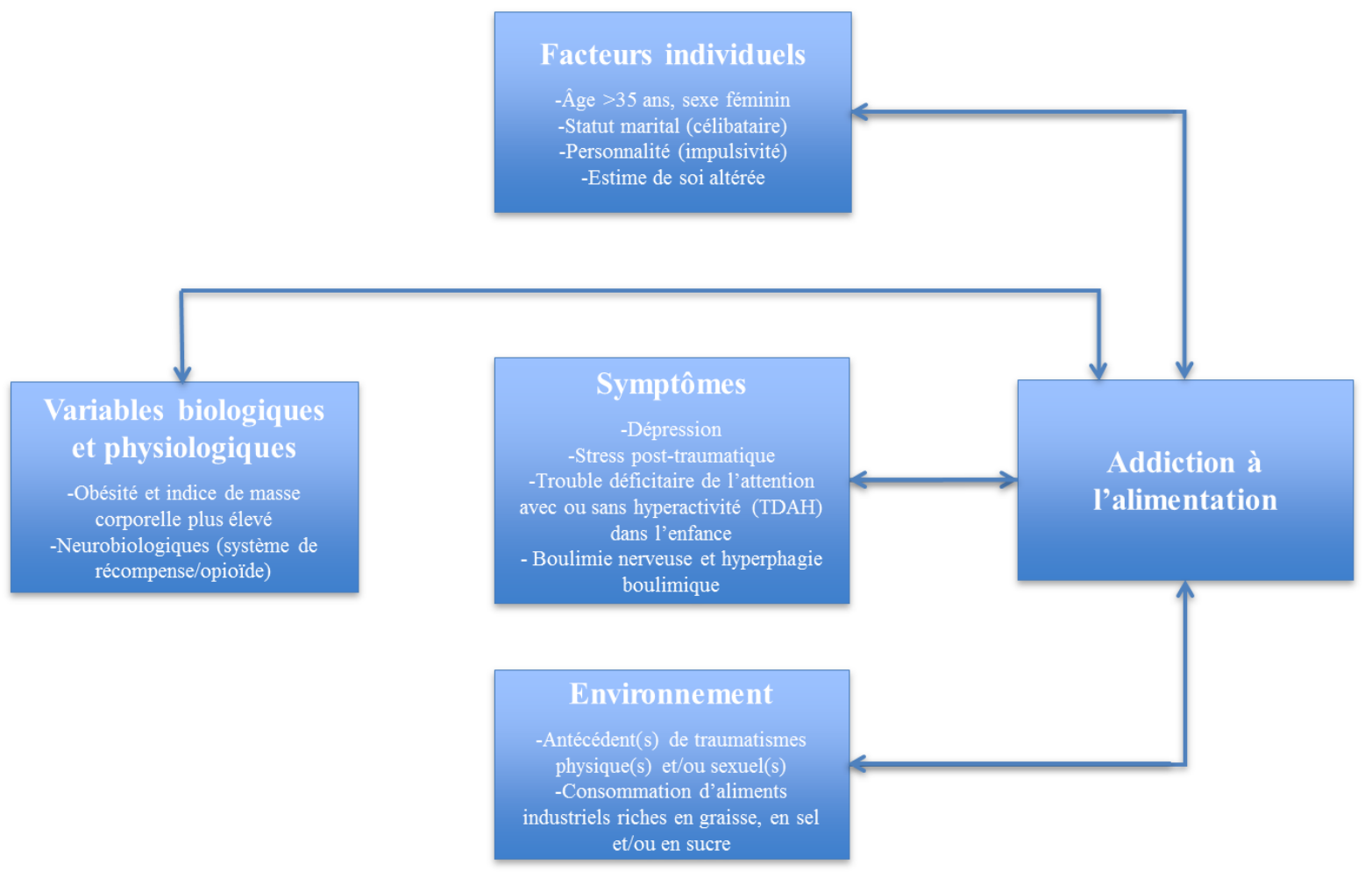

